\title{
William-Beuren Syndrome: Comprehensive Dental Care in Child Patient
}

\author{
Naila Marzuqi, DDS, ${ }^{1}$ Aulia Nuur Ainayah, DDS, ${ }^{1}$ Amalia Ramadhani Mufida, DDS, ${ }^{1}$ Nadhia Zahria Fajrin, DDS, ${ }^{1}$ \\ Tania Saskianti, DDS, $\mathrm{PhD}^{1}$ and Andra Rizqiawan, DDS, $\mathrm{PhD}^{2}$ \\ ${ }^{1}$ Pediatric Dentistry Department, Faculty of Dental Medicine, Universitas Airlangga, East Java, Surabaya, Indonesia \\ ${ }^{2}$ Oral Maxillofacial Surgery Department, Faculty of Dental Medicine, Universitas Airlangga, East Java, Surabaya, Indonesia
}

\begin{abstract}
The most common symptoms of Williams-Beuren syndrome (WBS) are heart defects and unusual facial features. The objective of this case report was to highlight the preparation of comprehensive dental care for this syndrome. A seven-year-old boy came with multiple dental caries as a chief complaint. Parents reported a history of corrected tetralogy of Fallot (ToF) three years ago. The routine medical check-up was carried out annually. Based on the typical signs and symptoms, a diagnosis of William-Beuren Syndrome was made. Comprehensive dental care under general anesthesia on a patient with a history of heart defect and multiple dental caries was the best choice and exhibits a high success rate. Extraction treatment was carried out to avoid the risk of endocarditis infection, restoration to prevent the progressivity of caries, and space maintainers to restore function and aesthetics.
\end{abstract}

Keywords: child, dental caries, William-Beuren Syndrome

\section{INTRODUCTION}

William-Beuren Syndrome (WBS) is a rare genetic syndrome with a multisystemic disorder, which has distinctive physical features and developmental abnormalities. ${ }^{1}$ The etiology is an autosomal dominant or new mutation. ${ }^{2}$ The prevalence is around 1 in 10,000 live births. Based on previous research, the Latin America population obtained a prevalence of $55 \%$ in male and diagnosed on 11.9 years old, African population obtained a prevalence of $75 \%$ in male and diagnosed on 7.7 years old, and Asian obtained a prevalence of $50 \%$ in male and diagnosed on 8 years old, unfortunately, there is no research regarding the prevalence in Indonesia. ${ }^{3}$

William-Beuren Syndrome manifestations including intellectual disability, hypersocial behavior, distinctive facial, short stature, connective tissue anomalies, endocrine abnormalities such as hypercalcemia, and cardiovascular disease. ${ }^{3-5}$

Craniofacial characteristics of children with WilliamBeuren syndrome including a broad forehead, bitemporal narrowing, narrow nasal bridge, periorbital fulness, malar flattening, long philtrum, wide mouth, thick lips vermillion, and prominent earlobes, deficient chin bone and micrognathia, and oral manifestation include malformed

Corresponding author: Tania Saskianti, DDS, PhD

Department of Pediatric Dentistry

Faculty of Dental Medicine

Universitas Airlangga

Surabaya, Indonesia

Email: tania-s@fkg.unair.ac.id teeth, enamel hypoplasia, excessive interdental spacing, high arched palate, high prevalence of dental caries. ${ }^{6,7}$

Dental manifestations in this syndrome are malocclusion, enamel hypoplasia, malformed teeth, and dental caries. Enamel hypoplasia is the one of dental manifestations in 

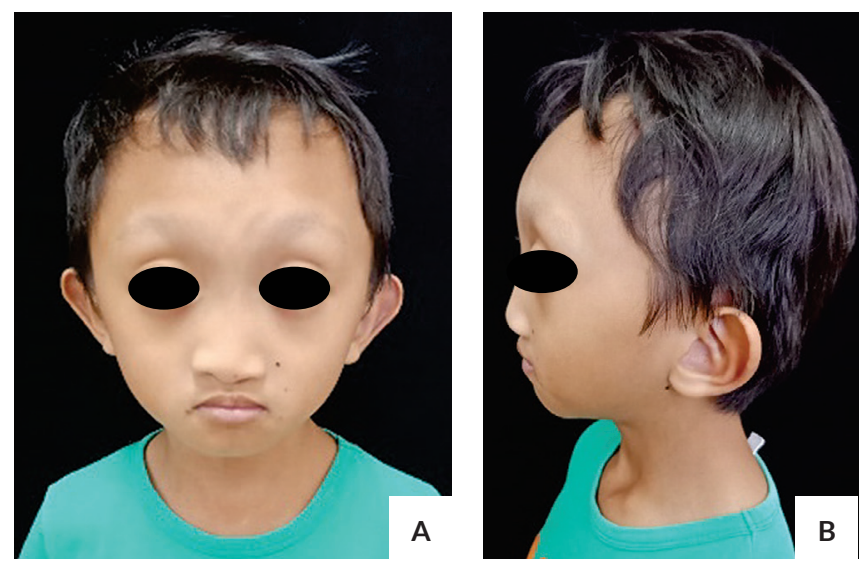

Figure 1. Facial characteristics (A) broad forehead, epichantal fold, flat nasal bridge, periorbital fullness, malar flattening, smooth philtrum, thick lips vermilion, small chin; (B) large earlobes.
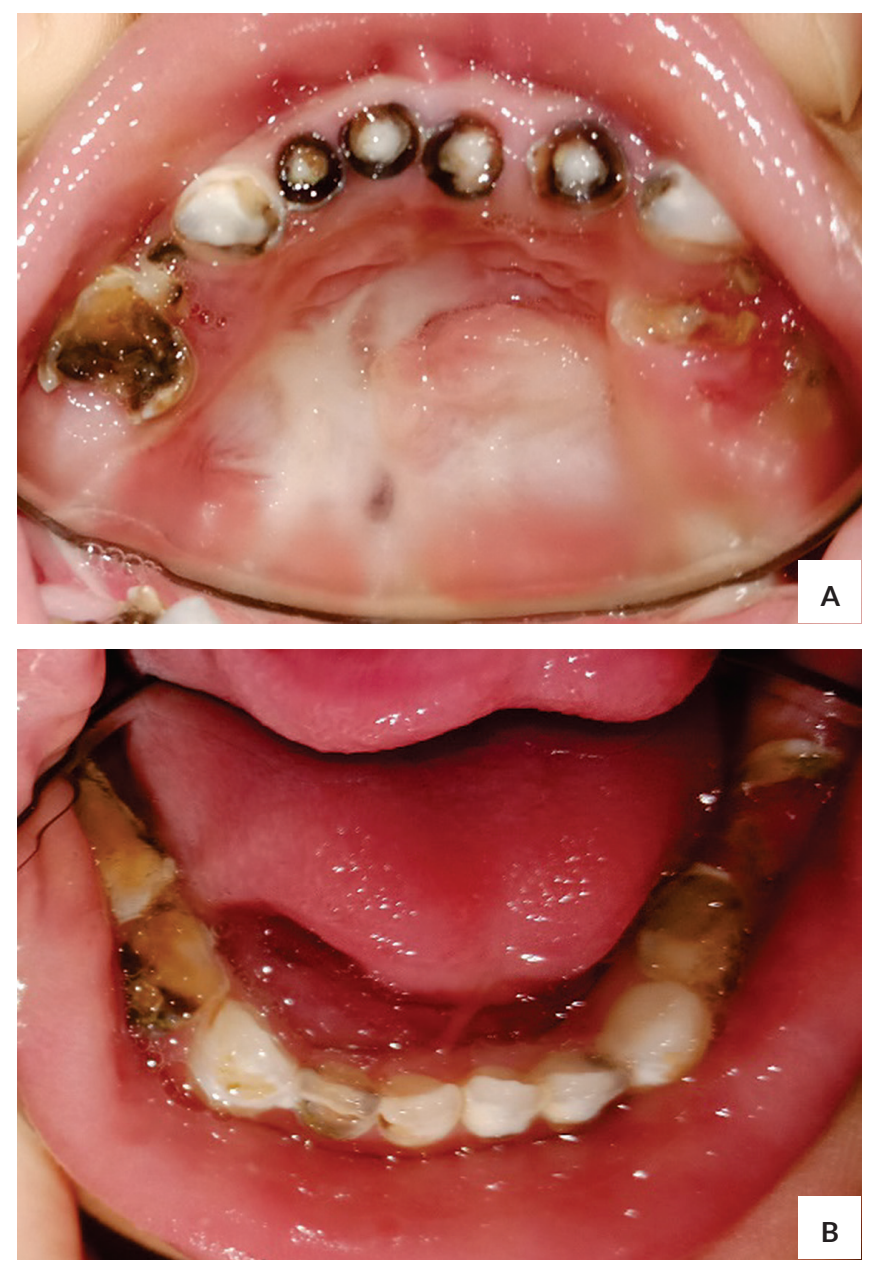

Figure 2. Intraoral examination before treatment $(\mathrm{A})$ the maxilla arch revealed multiple dental caries of 55,54 , $53,52,51,61,62,63,64,65$ and cleft palate (B) the mandibular arch revealed multiple dental caries of 75, 74, 73, 72, 71, 81, 82, 83, 84, 85.
WBS which increased the risk of dental caries. Since the parents focus on their child's medical treatment it caused less attention to oral hygiene. Dental caries was the most common oral disorder. Based on World Health Organization's data, dental caries emerges as one of the oral disorders leading to a person's lower life quality, both aesthetically and functionally. ${ }^{8}$

Dental treatment can be managed with behavior management which serves as one of the techniques employed to treat children with special needs. In a case where some treatments, e.g., administering sedatives, do not result in improvement, general anesthesia could be used as a therapeutic option to provide safe and effective dental treatment. ${ }^{9}$ Pediatric dentists can make a dental treatment plan with behavior modification and dental rehabilitation. ${ }^{10}$

Dental treatments in a medically compromised patient that aims to prevent and protect psychological development and reduce medical risks are one of the indications of general anesthesia. ${ }^{11}$ Dental treatment under general anesthesia for children with special needs could improve dental treatment quality, particularly preventing children from stress. For children with cyanotic heart defects, dental treatment may lead to stress, which may induce hypoxia, hyperpnea, and irritability. In this case, general anesthesia prevents the patient's stress due to multiple dental visits. ${ }^{12}$

In this case, general anesthesia was performed for dental treatment because somatic and emotional stress, such as pain, fear, excitement, and anxiety caused by the conventional dental treatment can lead to cardiovascular exacerbation. ${ }^{13}$

The treatment should be performed comprehensively by involving pediatric dentists, oral surgeons, cardiologists, anesthesiologists, and pediatricians to determine treatment procedures, prevent infective endocarditis, and improve dental treatment quality. ${ }^{12}$

\section{CASE REPORT}

A seven-year-old boy visited the Pediatric Dentistry of Universitas Airlangga's Dental Hospital with multiple dental caries as a chief complaint. The parents wanted to have their

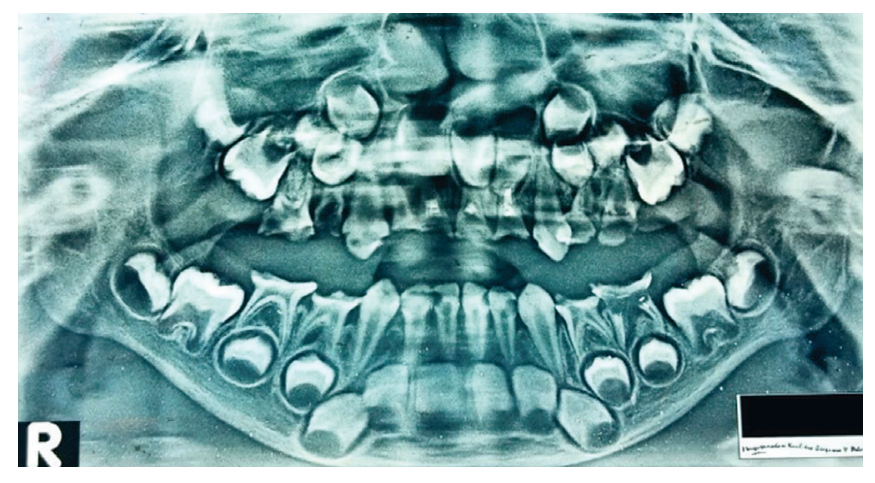

Figure 3. Orthopantomograph examination: tooth buds of permanent teeth, multiple dental caries of the maxilla and mandibular arch, and cleft palate. 
son's teeth treated to improve his appearance. The boy has a history of tetralogy of Fallot (ToF) three years ago and had been corrected. The patient's mother was infected by Rubella during pregnancy. Based on the typical signs and symptoms, a diagnosis of William-Beuren Syndrome was made by his pediatrician.

Intraoral examination showed multiple dental caries. The diagnosis included pulpitis reversible 53, 63, 71, 73, 83, 84, 85 , pulpitis irreversible $72,74,81,82$, gangrene radix 55,54 , $52,51,61,62,64,65,75$ and post surgery relapse of cleft palate surgery 5 years ago.

\section{CASE MANAGEMENT}

Comprehensive dental care was performed under general anesthesia after the patient received the complete examination from the pediatrician, anesthesiologist, and cardiologist. The patient had a complete blood count, urine test and admitted to our hospital the day before surgery.
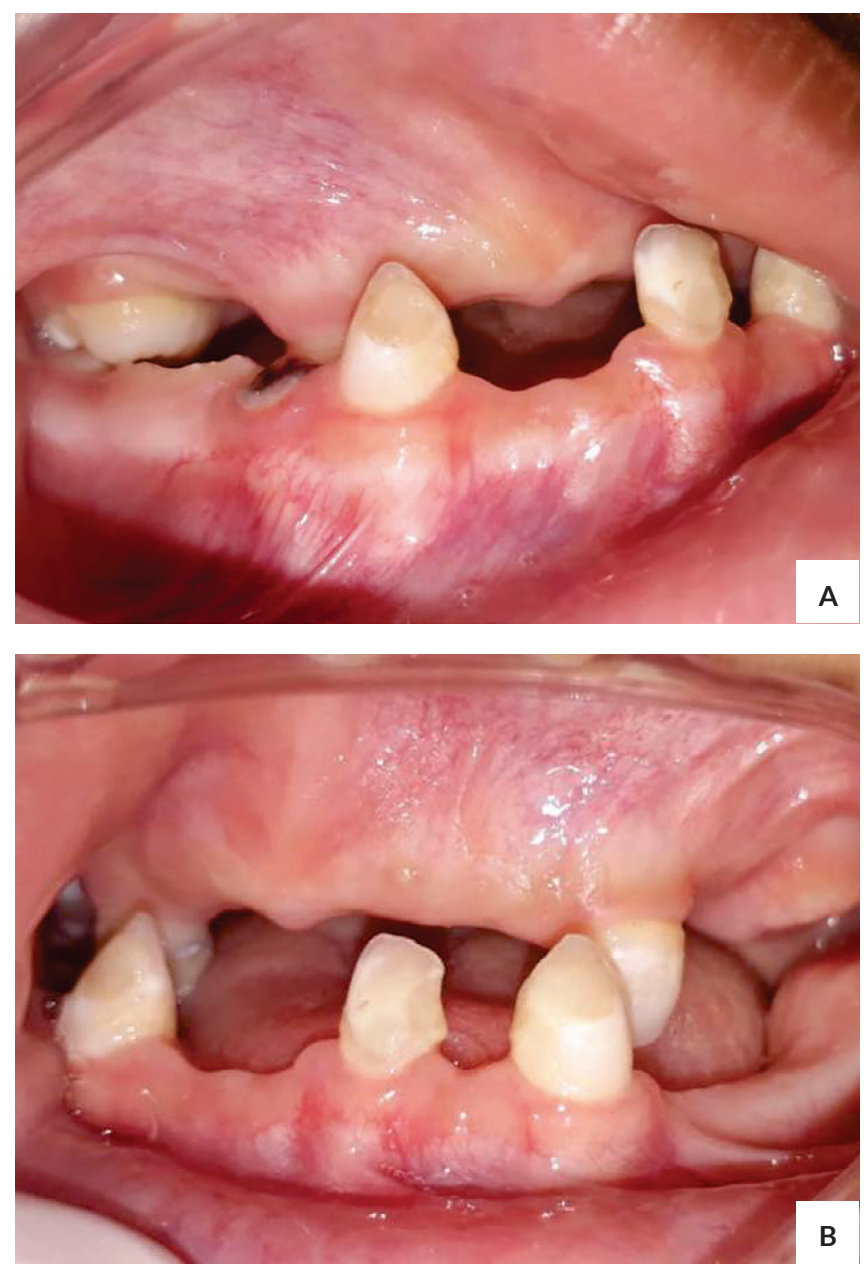

Figure 4. One month after the surgery, good wound healing (A) the left side: GIC restoration 63, 73, 71 remain intact; (B) the right side: GIC restoration 53, 83 remain intact.
The parents were informed about the treatment procedure, and informed consent was obtained. Dental treatment was performed after obtaining approval from his cardiologist, pediatrician, and anesthesiologist.

The treatment began by administering general anesthesia to the patient, the dental treatment was carried out by a pediatric dentist and oral surgeon. The dental treatment includes GIC restoration on tooth $53,63,71,73,83$, occlusal grinding and polishing on tooth 84,85 , and application of topical fluoride exercised by the pediatric dentist. Treatment was continued by oral surgeon to extract tooth $55,54,52,51$, $61,62,64,65,75,72,74,81,82$ and suturing. Cleft palate was not corrected according to the parents' informed consent.

Two weeks after the surgery, the patient came to remove the suture. A month after surgery, the patient was given partial dentures as the space maintainer. A partial denture was fabricated to improve oral, aesthetic, and mastication functions, thus increasing the patient's self-confidence.
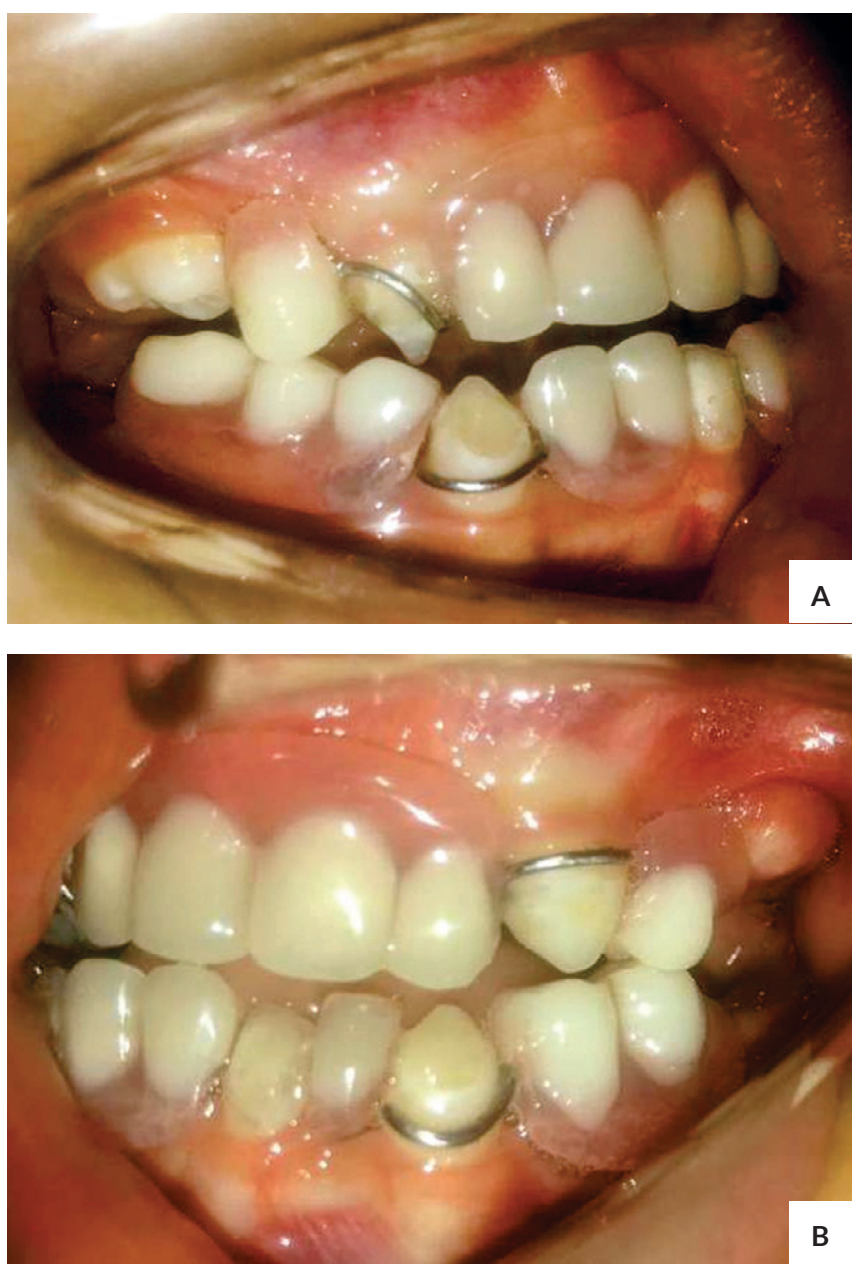

Figure 5. Insertion of the partial denture as space maintainer (A) the right side; (B) the left side. 


\section{DISCUSSION}

The patient was diagnosed William-Beuren Syndrome, a rare neurodevelopmental disorder that leads to certain clinical, behavioral, and cognitive profiles. It is a multisystemic condition that is caused by hemizygous deletion of 1.5 to $1.8 \mathrm{Mb}$ on chromosome $7 \mathrm{q} 11.23 .4,5$

Some manifestation including cardiovascular disease, short stature, broad forehead, epichantal fold, flat nasal bridge, periorbital fullness, malar flattening, smooth philtrum, thick lips vermilion, small chin (Figure 1A), large earlobes (Figure 1B), congenital heart disease (Tetralogy of Fallot), and multiple dental caries (Figures 2A, 2B and 3) were seen in this patient but not for intellectual disability. ${ }^{3,14}$

Enamel hypoplasia is the one of dental manifestations in WBS which can increase the risk of dental caries, and the parents' focus of medical treatment on patients can cause less attention to oral hygiene. ${ }^{6,7}$

In another case reported, a 28-month-old boy diagnosed with WBS with cardiovascular disease received dental treatment under pharmacological behavior management (general anesthesia). Somatic and emotional stress, such as pain, fear, excitement, and anxiety caused by the conventional dental treatment could lead to a cardiovascular exacerbation. Curative dental treatment was also necessary because dental caries could pose a risk to endocarditis due to focal infections. ${ }^{13}$

A medical evaluation to rule out cardiovascular abnormalities which could affect dental treatment is mandatory. Cases of sudden death have been reported with the administration of anesthetic in these individuals. Hence, caution should be exercised while administering anesthesia in these individuals, but in this case, performed treatment under general anesthesia was chosen because many treatments must be done more efficiently in one visit to reduce patient anxiety and increase dental treatment quality. ${ }^{1}$

General anesthesia could be applied to patients who are considered non-cooperative due to physical, mental, or medical disorders, young patients, patients with cognitive or emotional immaturity who tend to have extreme anxiety ad requires extensive rehabilitation, non-cooperative behaviors, patients requiring extensive treatment, patients who need tooth restoration or surgery that should be performed simultaneously. ${ }^{11,15,16}$

GIC restorations (Figure 4) were chosen because of the simple handling technique and fluor release (the ion exchanges between glass-ionomer cement and dentine facilitate the remineralization of caries-affected dentine into fluorapatite that provides a caries-resistant). Root canal treatment was not recommended in this case due to the high incidence of chronic infections. Tooth extraction ended with suturing to ensure wound healing. The utilization of sutures in attempting primary wound closure and the role of suturing in providing wound stabilization. ${ }^{17,18}$
A removable partial denture (Figure 5) as a space maintainer was fabricated to maintain the space of the dental arch, fill the physiological space, and guidance of the permanent tooth eruption. ${ }^{19}$

Treatment was carried out in one visit and comprehensive with a multidisciplinary team (cardiologist, anesthesiologist, oral surgeon, pediatric dentist, and pediatrician). Extraction, restoration, occlusal grinding was performed in this case. It can reduce the progression of caries, improve aesthetics, function and increase self-confidence.

\section{CONCLUSION}

Regarding various considerations, comprehensive dental treatment for William-Beuren syndrome in one visit was deemed suitable. General anesthesia was chosen as appropriate behavior management pharmacologically. Comprehensive care is the best option to make treatment plans for patients with William-Beuren syndrome.

\section{Statement of Authorship}

All authors participated in data collection and analysis, and approved the final version submitted.

\section{Author Disclosure}

All authors declared no conflicts of interest.

\section{Funding Source}

No funding support.

\section{REFERENCES}

1. Wong D, Ramachandra, Sulugodu S, Singh AK. Dental management of patient with Williams Syndrome - A case report. Contemp Clin Dent. 2015;6(July):418-20.

2. Paul S. Casamassimo, Fields HW, McTigue DJ, Nowak AJ. Pediatric Dentistry Infancy through Adolescence. 5 ed. St. Louis, Missouri: Elsevier Inc; 2013.

3. Kruszka P, Porras AR, de Souza DH, Moresco A, Huckstadt V, Gill $\mathrm{AD}$, et al. Williams-Beuren syndrome in diverse populations. Am J Med Genet Part A. 2018;176(5):1128-36.

4. De Lorenzo F, Macchiaiolo M, Carlevaris CM, Bartuli A. The work experience of a patient affected by Williams Syndrome: a pilot project at the Bambino Gesù Children's Hospital. Orphanet J Rare Dis. 2017;12(1):10-3.

5. Van Herwegen J. Williams syndrome and its cognitive profile: The importance of eye movements. Psychol Res Behav Manag. 2015; 8:143-51.

6. Li J, Zhang H, Yang C, Li Y, Dai Z. An overview of osteocalcin progress. J Bone Miner Metab. 2016;34(4):367-79.

7. Ferreira SBP, Viana MM, Maia NGF, Leão LL, Machado RA, Coletta $\mathrm{R}$ Della, et al. Oral findings in Williams-Beuren syndrome. Med Oral Patol Oral y Cir Bucal. 2018;23(1):e1-6.

8. Yadav K, Prakash S. Dental Caries: A Review. Asian J Biomed Pharm Sci. 2016;53(6):1-7.

9. Ferrazzano GF, Salerno C, Sangianantoni G, Caruso S, Ingenito A, Cantile T. The effect of dental treatment under general anesthesia on quality of life and growth and blood chemistry parameters in uncooperative pediatric patients with compromised oral health: A pilot study. Int J Environ Res Public Health. 2020;17(12):1-16. 
10. Patil P, Arora G, Poornima P, Subbareddy V. Dentofacial characteristics in William's syndrome. Contemp Clin Dent. 2012;3(5):41.

11. Sharma A, Jayaprakash R, Babu NA, Masthan KMK. General anaesthesia in pediatric dentistry. Biomed Pharmacol J. 2015;8SE (June 2018):189-94.

12. Mahalingam A, Vaidyanathan K, Ilango P, Cherian S. Dental Management Of A Child With Congenital Heart Disease - A Case Report. Int J Recent Sci Res. 2019;10(C):30830-3.

13. Kohase H, Wakita R, Doi S, Umino M. General anesthesia for dental treatment in a Williams syndrome patient with severe aortic and pulmonary valve stenosis: suspected episode of postoperatively malignant hyperthermia. Oral Surgery, Oral Med Oral Pathol Oral Radiol Endodontology. 2007;104(4):17-20.

14. Cogulu D, Hazan F, Cagirir FD. Orofacial findings and dental management of Williams syndrome. Genet Couns. 2015;26(4):437-42.
15. Ramazani N. Different aspects of general anesthesia in pediatric dentistry: A review. Iran J Pediatr. 2016;26(2).

16. Silva CC, Lavado C, Areias C, Mourão J, Andrade D de. Conscious sedation vs general anesthesia in pediatric dentistry - a review. Med Express. 2015;2(1):1-4.

17. Nelson WJ. Guide to Suturing. J oral Maxillofac Surg. 2015;73(8):4-5.

18. Knight GM. The benefits and limitations of glass-ionomer cements and their use in contemporary dentistry. In: Glass-Ionomers in Dentistry [Internet]. Springer International Publishing; 2016 [cited $2021 \mathrm{Feb}$ 10]. p. 57-79. Available from: https://link.springer.com/ chapter/10.1007/978-3-319-22626-2_3

19. Khare V, Nayak PA, Khandelwal V, Nayak UA. Fixed functional space maintainer: novel aesthetic approach for missing maxillary primary anterior teeth. BMJ Cse Rep. 2013;1-4.

Have you read the current trends in Medical and Health Research in the Philippines?

\title{
Acta Medica Philippina The National Health Science Journal
}

\author{
Access Online: www.actamedicaphilippina.upm.edu.ph
}

